EPJ Web of Conferences 21, 09003 (2012)

DOI: $10.1051 /$ epjconf/20122109003

(C) Owned by the authors, published by EDP Sciences, 2012

\title{
Clustering Pre-equilibrium Model Analysis for Nucleon-induced Alpha-particle Spectra up to $200 \mathrm{MeV}$
}

\author{
S. Kunieda ${ }^{1,2, a}$, T. Kawano ${ }^{1}$, M. B. Chadwick ${ }^{1}$, T. Fukahori ${ }^{2}$, and Y. Watanabe ${ }^{3}$ \\ 1 Theoretical Division, Los Alamos National Laboratory, Los Alamos, New Mexico 87545, USA \\ 2 Nuclear Data Center, Japan Atomic Energy Agency, Tokai-mura Naka-gun, Ibaraki 319-1195, Japan \\ 3 Department of Advanced Energy Engineering Science, Kyushu University, Kasuga, Fukuoka, 816- \\ 8580, Japan
}

\begin{abstract}
The clustering exciton model of Iwamoto and Harada is applied to the analysis of pre-equilibrium $(N, x \alpha)$ energy spectra for medium-to-heavy nuclei up to $200 \mathrm{MeV}$. In this work, we calculate alpha-particle formation factors without any approximations that appear in the original model. The clustering process is also considered in both the primary and second pre-equilibrium emissions. We optimize the exciton and the clustering model parameters simultaneously by looking at the experimental $(N, x N)$ and $(N, x \alpha)$ energy spectra. The experimental alpha-particle spectra are well reproduced with a unique set of clustering model parameters, which is independent of incident neutrons/protons. The present analysis also implies that the clustering model parameter is not so different between the medium and heavy nuclei. Our calculations reproduce experimental data generally well up to the incident energy of $\sim 150 \mathrm{MeV}$, but underestimations are seen above this energy.
\end{abstract}

\section{Introduction}

The exciton model, as it is frequently used in the nuclear data evaluation [1], predicts pre-equilibrium $(N, x N)$ spectra generally well. However, a long-standing problem exists in the model calculation of the pre-equilibrium composite-particle spectra due to rather complicated reaction mechanism. The phenomenological models proposed by Kalbach [2,3] describe nucleon-transfer reaction process in the phase-space, in which many adjustable parameters are involved to fit experimental particle energyspectra. Although a useful global parameterization is reported [2,3], extrapolation of the global parameters beyond the experimental range needs much attention.

We apply the clustering exciton model of Iwamoto and Harada [4] to calculate nucleon-induced pre-equilibrium alpha-particle spectra from medium-to-heavy nuclei. The model describes the pickup process within the exciton model framework, where the alpha-particle formation factor is calculated by the overlap integral of wave functions between the alpha-particle and four nucleons (include the nucleons below Fermi level). In this work, the formation factors are calculated by numerical integrations, while the original Iwamoto-Harada model employed the root-mean-square approximation [4] where no correlation exists between the coordinates in the phase-space. The clustering process is also considered in both the primary and second pre-equilibrium emissions. We optimize the pre-equilibrium and the clustering model parameters simultaneously by looking at the experimental nucleon and alphaparticle energy spectra.

In this paper, differences between the exact calculation and the approximation are exhibited for the alpha-particle formation factor. Through the comparisons with experimental spectra at incident energies up to $\sim 200 \mathrm{MeV}$, we show the behavior of the clustering model parameter, the applicability and limitation of the model.

\footnotetext{
a e-mail: kunieda.satoshi@jaea.go.jp
}

This is an Open Access article distributed under the terms of the Creative Commons Attribution-Noncommercial License 3.0, which permits unrestricted use, distribution, and reproduction in any noncommercial medium, provided the original work is properly cited. 


\section{EPJ Web of Conferences}

\section{Model Framework}

The idea of clustering exciton model was originally proposed by Iwamoto and Harada [4], in which the alpha-particle formation factors are calculated from the overlap integral between the wave functions of alpha-particle and four nucleons. It is symbolically expressed as $\left\langle\varphi_{\alpha} \chi^{\left(\epsilon_{\alpha}\right)}(\mathbf{R}) \mid \phi_{1} \phi_{2} \phi_{3} \phi_{4}\right\rangle$ where $\varphi_{\alpha}$ and $\chi^{\left(\epsilon_{\alpha}\right)}(\mathbf{R})$ denote the intrinsic and the center-of-mass wave functions of alpha-particle, $\phi_{1, \ldots, 4}$ the wave functions of the single-particles. Numerically, the formation factor is calculated by the multiple integrations over the phase-space,

$$
F_{l, m}\left(\epsilon_{\alpha}\right)=\frac{1}{(2 \pi \hbar)^{9}} \int_{S} \prod_{i=1}^{3} d \xi_{\mathrm{i}} d \mathbf{p}_{\xi_{\mathrm{i}}}
$$

where the coordinates $\left(\xi_{\mathrm{i}}, \mathbf{p}_{\xi_{\mathrm{i}}}\right)_{i=1,2,3}$ are introduced to describe relative motions of two $N$ - $N$ and one $2 N-2 N$ systems. The integration ranges are determined by the ground-state Hamiltonian of alphaparticle under the conditions $p_{i=(1, \ldots, l)} \geq p_{f}, p_{j(=l+1, \ldots, 4)}<p_{f}$ and $r_{i(=1, \ldots, 4)} \leq R_{r e s}+\Delta R$, where $p_{f}$ is the strength of Fermi momentum, $R_{\text {res }}$ the radius of the residual nuclei. The symbol $\Delta R$ physically means the nuclear surface area where pickup reaction may occur. This is a major parameter of this model, and determine the overall behavior of the formation factors. In this work, the formation factors are exactly calculated by numerical integrations, while the original Iwamoto-Harada model employed the root-mean-square approximation [4]. Figure 1 illustrates the differences between the exact calculation and the rms approximation for $F_{1,3}, F_{2,2}, F_{3,1}$ and $F_{4,0}$. Those calculations were made for for $p+{ }^{40} \mathrm{Ca}$ system under the condition of $\Delta R=1.0 \mathrm{fm}$, which was assumed in the original work. The exact and approximated calculations exhibit the same behavior, but the absolute values are apparently different. One also notices that the high-energy tail lingers in the exact calculation, while it suddenly drops to zero at rather lower energies in the approximation. In our data analysis, we consistently use the exact calculations of Eq. (1), which avoids any deficiencies coming from the rms approximation.

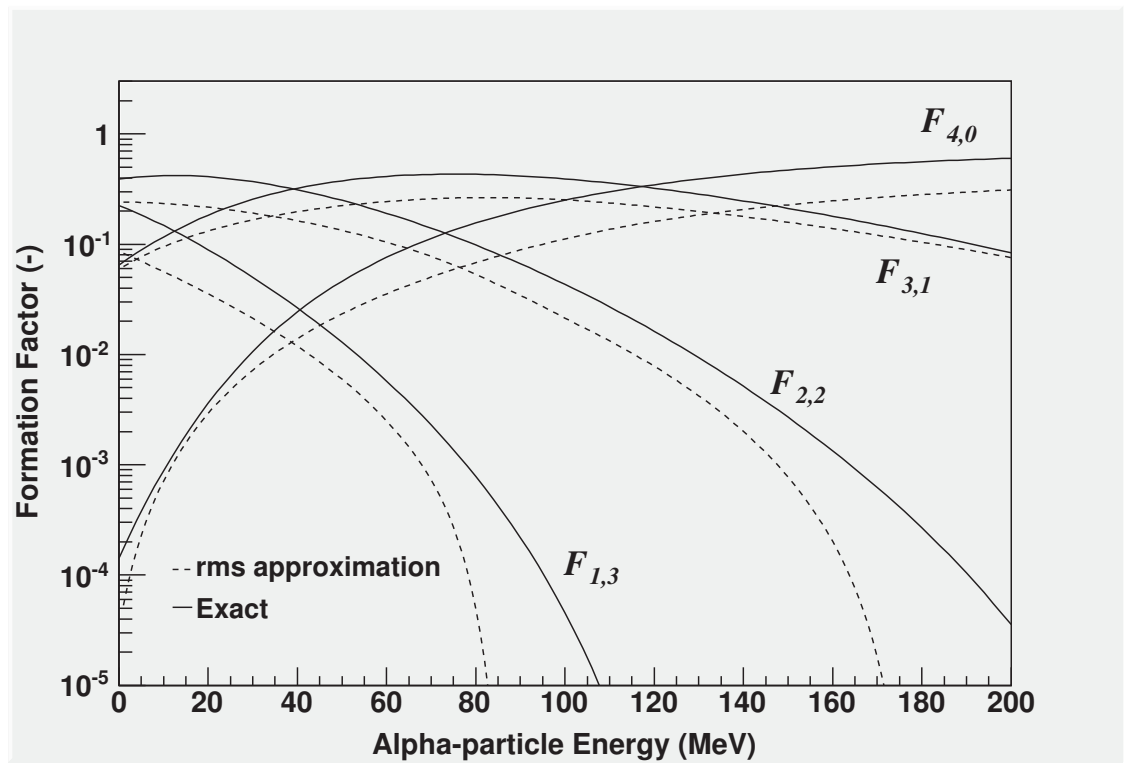

Fig. 1. The formation factors of alpha-particle $F_{l, m}$ for $p+{ }^{40} \mathrm{Ca}$ system under the condition of $\Delta R=1.0 \mathrm{fm}$

The cross sections are calculated with the Hauser-Feshbach code GNASH [5] in which the exciton model is adopted for pre-equilibrium process. The primary nucleon emission rates are calculated allowing those from the auxiliary configurations [2]. Similarly, we modify the code to compute the 


\section{$\mathrm{CNR} * 11$}

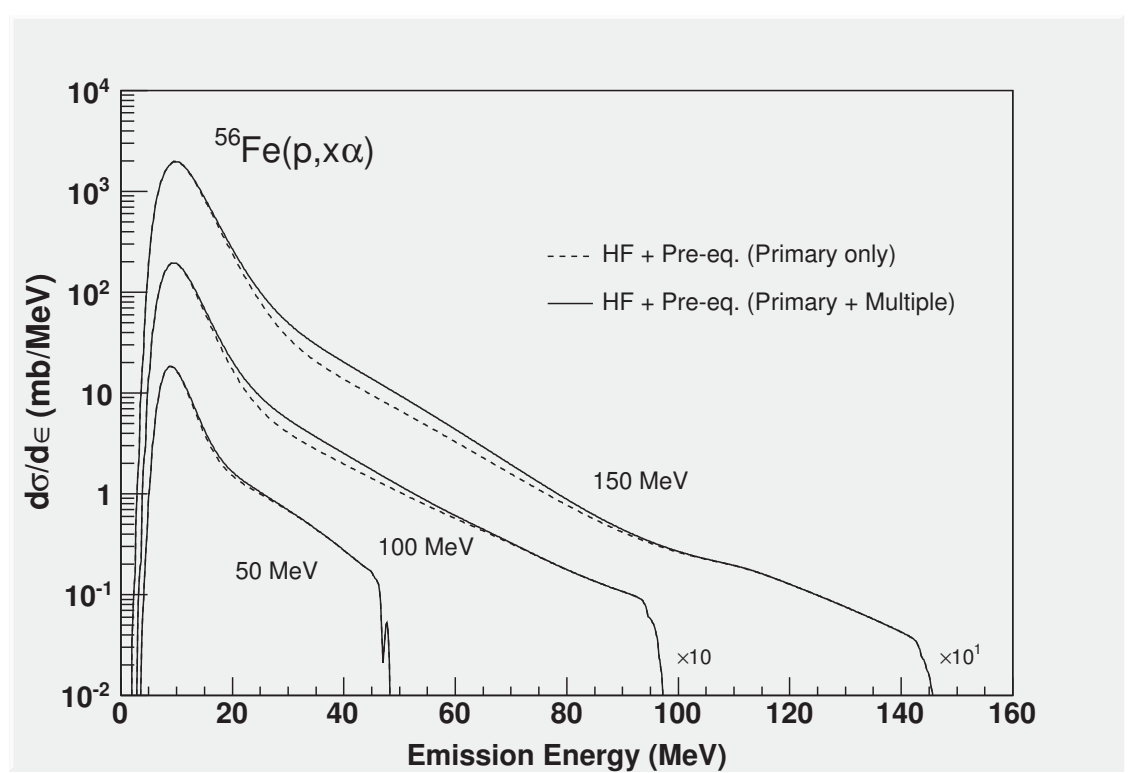

Fig. 2. Calculated angle-integrated spectra for ${ }^{56} \mathrm{Fe}(p, x \alpha)$ at the incident energies of 50, 100 and $150 \mathrm{MeV}$. The solid and dashed curves represent the total alpha-particle spectra with and without the multiple pre-equilibrium emission, respectively.

primary emission rate of alpha-particle by introducing the formation factor $F_{l, m}\left(\epsilon_{\alpha}\right)$ as

$$
W_{\alpha}\left(p, h, \epsilon_{\alpha}\right)=\frac{1}{\pi^{2} \hbar^{3}} \mu_{\alpha} \epsilon_{\alpha} \sigma_{\alpha} \frac{\sum_{l+m=4} F_{l, m}\left(\epsilon_{\alpha}\right)\left[\sum_{j=0}^{2} \omega(p-l, h-j, U)+\sum_{j=1}^{2} \omega(p-l-j, h, U)\right]}{\sum_{j=0}^{2} \omega(p, h-j, E)+\sum_{j=1}^{2} \omega(p-j, h, E)} .
$$

The symbol $\sigma_{\alpha}$ denotes the inverse reaction cross sections which is calculated by the optical model, and $\mu_{\alpha}$ stands for the reduced mass. The single-particle state density $\omega(p, h, E)$ is given with the finite well depth correction [6] as $\omega(p, h, E, \infty) f(p, h, E, V)$. We also made it possible to calculate alpha-particle emission from the excited residual nuclei that were produced by the primary $(N, N)$ pre-equilibrium reaction. The multiple particle emission spectra through such processes are calculated as in Ref. [7] by considering the clustering. Figure 2 represents those model calculations, in which alpha-particle spectra are illustrated for ${ }^{56} \mathrm{Fe}$ at the incident proton energies of 50, 100 and $150 \mathrm{MeV}$. The multiple emissions enhance the lower energy part of spectra above the incident energy of $\sim 50 \mathrm{MeV}$ as plotted in this figure.

\section{Model Parameters}

The pre-equilibrium model calculation requires total reaction cross sections for incident particle, and inverse reaction cross sections for outgoing particles. In this work, those values are calculated with the optical model. The coupled-channles calculations were performed for nucleons with a local/global optical potential as in Ref. [8]. For alpha-particles, the spherical model calculations were made with the optical potential of Avrigeanu et al. $[9,10]$. Those calculations also provide transmission coefficients for outgoing particles that are used in the Hauser-Feshbach statistical model calculation.

In the exciton model, the effective potential well depth $V$ is taken from the global pre-equilibrium analysis of Koning and Duijvestijn [11]. The single-particle state density parameter is set as $g=6 a / \pi^{2}$, 


\section{EPJ Web of Conferences}

where the symbol $a$ is the level density parameter that is calculated in the expression of Ignatyuk et al. [12] (the asymptotic values are taken from those obtained by Arthur [5]). We evaluate the average matrix element $M^{2}$ of Kalbach [13] to fit the available experimental $(N, x N)$ pre-equilibrium spectra.

The ground-state Hamiltonian of alpha-particle is described by the harmonic-oscillator model in accordance with the original work of Iwamoto and Harada. The harmonic-oscillator parameter is chosen to give the rms of $1.6 \mathrm{fm}$, which is consistent with the experimental value. For the Fermi energy, we take $38 \mathrm{MeV}$ irrespective of nucleus. The radius of residual nuclei is set to $R_{\text {res }}=1.5 A^{1 / 3} \mathrm{fm}$ as assumed in the original work. We finally obtain the clustering parameter $\Delta R$ so as to give a good agreement with the available experimental $(N, x \alpha)$ spectra.

According to our $(n, x \alpha)$ analysis for ${ }^{59} \mathrm{Co}$ and the neighboring nuclei, reasonable spectra can be obtained with $\Delta R \sim 0.75 \mathrm{fm}$, once the average matrix element $M^{2}$ is well determined. Figure 3 represents this situation, in which the model calculations are made with $\Delta R=0.75 \mathrm{fm}$ and compared with the experimental data of Nica et al. [14] at the incident energy of $62.7 \mathrm{MeV}$ for ${ }^{59} \mathrm{Co}$. The calculations

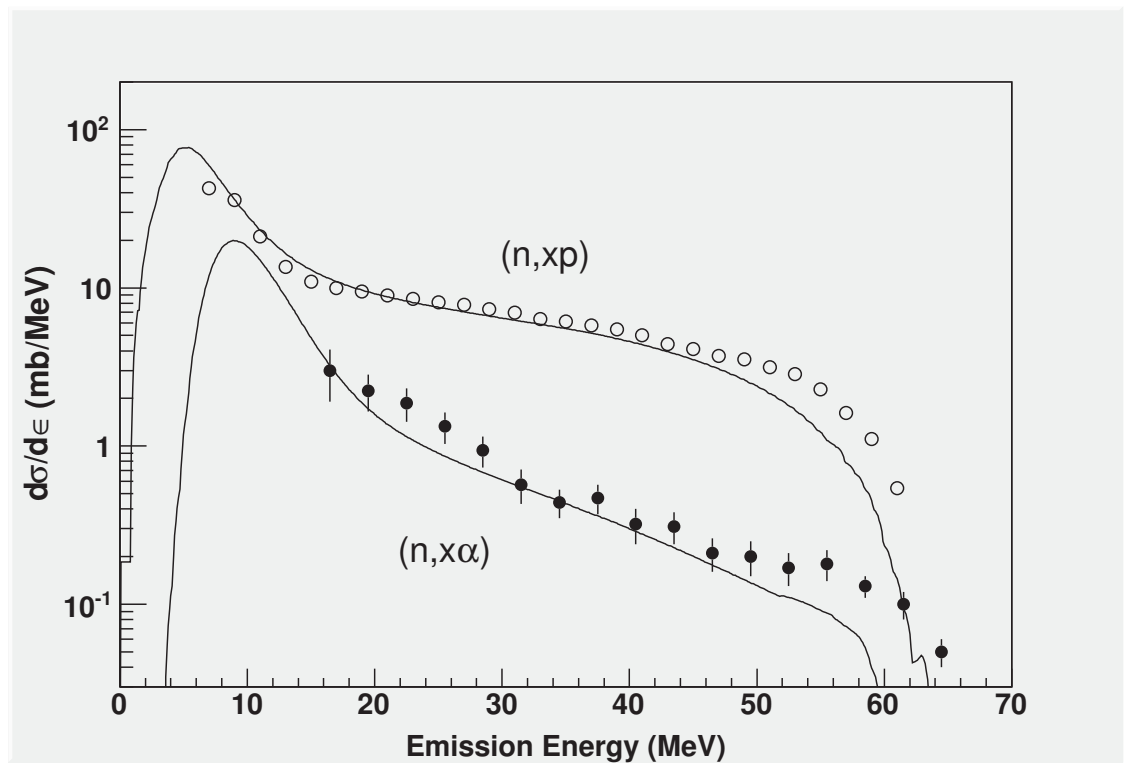

Fig. 3. Angle-integrated spectra for ${ }^{59} \mathrm{Co}(n, x p)$ and $(n, x \alpha)$ reactions at incident energy of $62.7 \mathrm{MeV}$ which are compared with the measured data of Nica et al. [14]

were also performed for proton-induced reaction for ${ }^{59} \mathrm{Co}$ and ${ }^{208} \mathrm{~Pb}$ assuming the same $\Delta R$ value. In Fig. 4 , the calculated $(p, x \alpha)$ spectra are compared with the experimental data of Lewandwski et al. [15] for ${ }^{59} \mathrm{Co}$ at $72.3 \mathrm{MeV}$ and ${ }^{208} \mathrm{~Pb}$ at $70.7 \mathrm{MeV}$, respectively. The present calculations reproduce the measured ${ }^{59} \mathrm{Co}(n, x \alpha)$ very well. Reasonable agreements are also seen even for the ${ }^{208} \mathrm{~Pb}$ case. According to those findings, the same $\Delta R$ value can be applied to both neutron- and proton-induced reactions. In addition, the parameter seems to be independent of the target mass.

\section{Applicability and Limitation}

Figure 5 illustrates the absolute $(p, x \alpha)$ cross sections for Fe from the threshold energy to $200 \mathrm{MeV}$. Our calculated cross sections agree with the experimental data of Ammon et al. [16] fairly well. For Fe, also it is true for other nuclei in this mass region, the statistical decay process gives major contributions to the total alpha-particle production cross section, and the cross section becomes much larger than that from pre-equilibrium process. Nevertheless, the pre-equilibrium process is still important above 10 


\section{$\mathrm{CNR} * 11$}
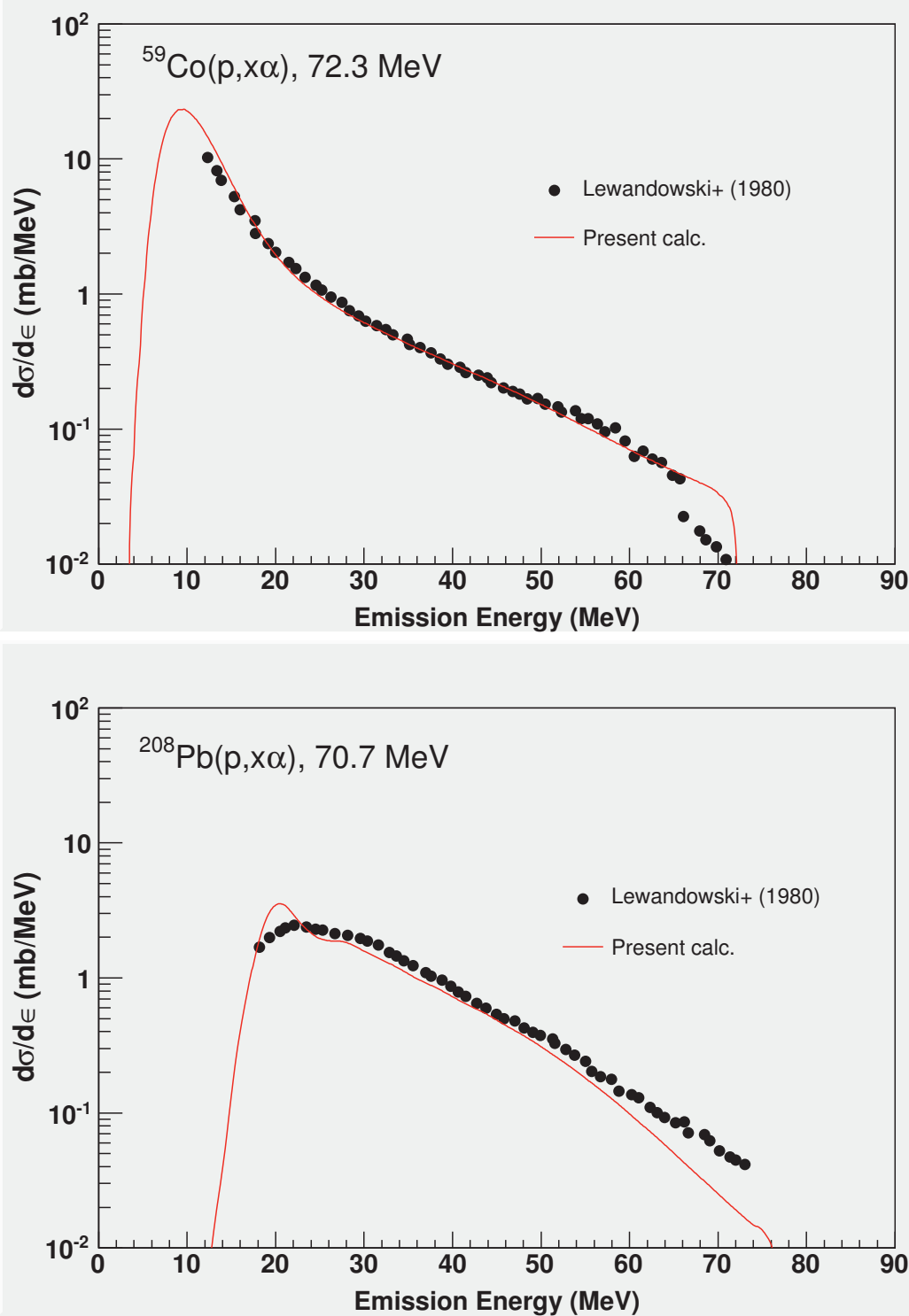

Fig. 4. Angle-integrated ( $p, x \alpha)$ spectra for ${ }^{59} \mathrm{Co}$ at $72.3 \mathrm{MeV}$ and ${ }^{208} \mathrm{~Pb}$ at $70.7 \mathrm{MeV}$ which are compared with the measured data of Lewandwski et al. [15]

$\mathrm{MeV}$ to give the total alpha-particle production cross sections that are consistent with the experimental data.

Through the comparison with experimental data, we found that our calculation gives reasonable alpha-particle spectra up to the incident energy of $\sim 150 \mathrm{MeV}$. However, the situation is somewhat different above this energy. Figure 6 shows double differential cross sections for ${ }^{58} \mathrm{Ni}(p, x p)$ and $(p, x \alpha)$ reactions at the incident energy of $175 \mathrm{MeV}$. In this figure, we compare our calculated results with the measured data of Piskor-ignatowicz et al. [17] at emission angles of $16^{\circ}, 65^{\circ}$ and $100^{\circ}$. The doubledifferential cross sections were calculated by supplementary use of the angular-distribution systematics of Kalbach [18]. Our calculations nicely reproduce the experimental $(p, x p)$ spectra, but underestimate the measured $(p, x \alpha)$ spectra at the forward angle. This underestimation tends to be larger as emission 


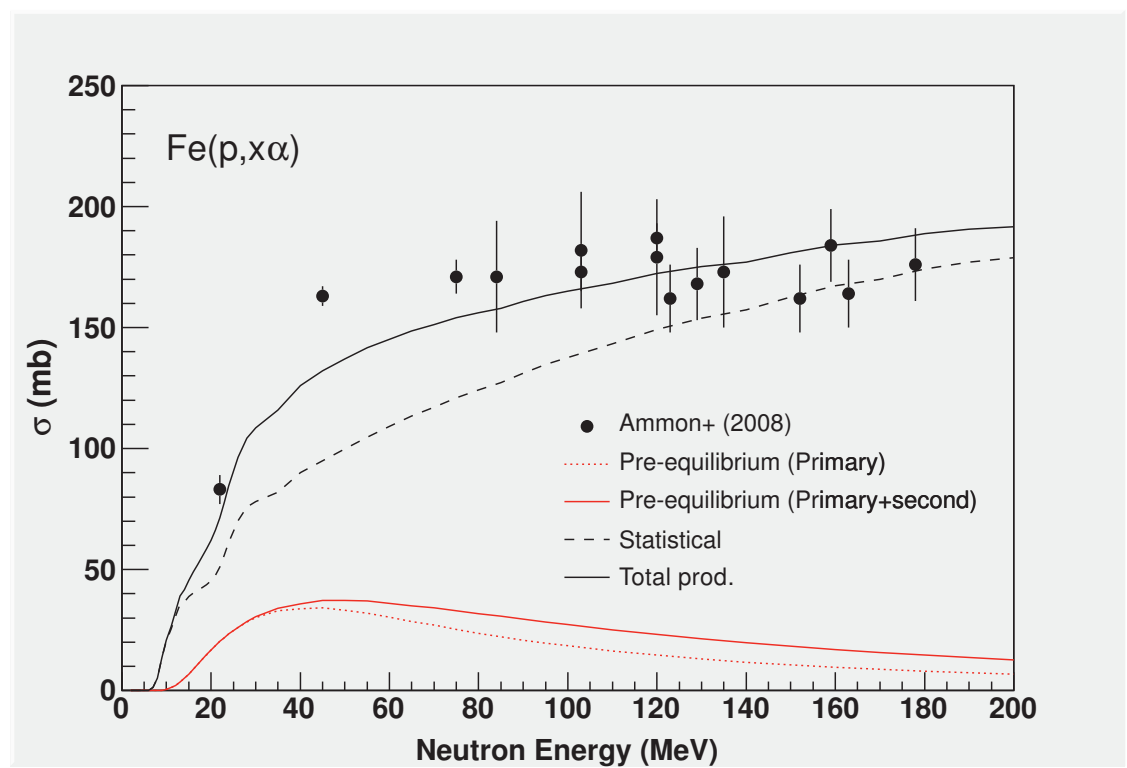

Fig. 5. Calculated $(p, x \alpha)$ cross sections for Fe from threshold energy to $200 \mathrm{MeV}$ which are compared with the experimental data of Ammon et al. [16]

energy increases. The same tendencies are also observed in comparisons with the measured data of Cowley et al. [19] who reported experimental $(p, x \alpha)$ spectra in the similar energy range for the other nuclei. At this moment, it is not so clear that this problem is due to applicability of the angular distribution systematics at very high energies, or due to other nuclear reaction mechanisms such as knock-out, or even both of them. Further works are needed to extend our modeling above $150 \mathrm{MeV}$.

\section{Summary and Conclusion}

We applied the clustering exciton model of Iwamoto and Harada to the analysis of pre-equilibrium $(N, x \alpha)$ spectra. The alpha-particle formation factors were calculated with the exact overlap-integral of wave functions, which gives much larger values than those calculated with the rms approximation. The clustering process is considered not only in the primary but also in the second pre-equilibrium emissions, which enhanced the lower energy spectra above the incident energy of $\sim 50 \mathrm{MeV}$. Once the exciton model parameters were determined, reasonable alpha-particle spectra can be obtained with a simple parameterization in the clustering model. According to the present analysis, the same $\Delta R$ value can be applied to both neutron- and proton-induced reactions. Also, the parameter does not seem to depend highly on the nucleus. Although our calculations reproduced experimental $(N, x \alpha)$ spectra up to $\sim 150 \mathrm{MeV}$, there still remained discrepancies in the energy range above this energy.

\section{Acknowledgments}

One of the authors S. Kunieda thanks Los Alamos National Laboratory for the hospitality during his stay. He is also grateful to Dr. S. Okajima of Japan Atomic Energy Agency who encouraged this study. This work was carried out under the auspices of the National Nuclear Security Administration of the U.S. Department of Energy at Los Alamos National Laboratory under Contract No. DE-AC5206NA25396. 

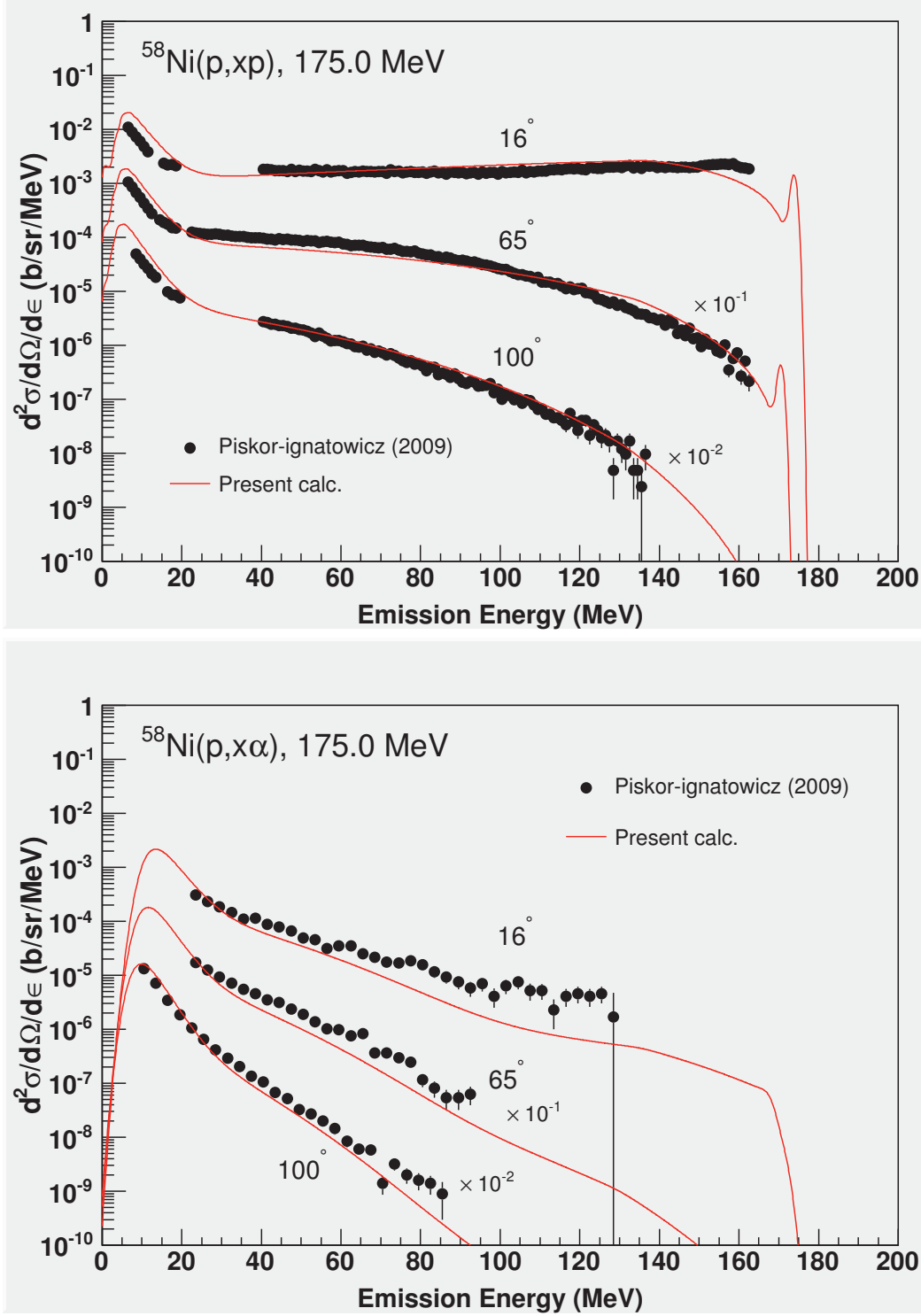

Fig. 6. Double differential cross sections for ${ }^{58} \mathrm{Ni}(p, x p)$ and $(p, x \alpha)$ reactions at incident energy of $175 \mathrm{MeV}$ which are compared with the measured data of Piskor-ignatowicz et al. [17]

\section{References}

1. M. B. Chadwick, P. Oblozinsky, M. Herman, N. M. Greene, R. D. McKnight and D. L. Smith, P. G. Young, R. E. MacFarlane, G. M. Hale, S. C. Frankle and A. C. Kahler, T. Kawano, R. C. Little, D. G. Madland, P. Moller, R. D. Mosteller, P. R. Page, P. Talou, H. Trellue, M. C. White, W. B. Wilson and R. Arcilla, C. L. Dunford, S. F. Mughabghab, B. Pritychenko, D. Rochman, A. A. Sonzogni, C. R. Lubitz, T. H. Trumbull, J. P. Weinman, D. A. Brown, D. E. Cullen, D. P. Heinrichs, D. P. McNabb, H. Derrien, M. E. Dunn, N. M. Larson, L. C. Leal, A. D. Carlson, R. C. Block, J. B. Briggs, E. T. Cheng, H. C. Huria, M. L. Zerkle, K. S. Kozier, A. Courcelle, V. Pronyaev and S. C. van der Marck, Nucl. Data Sheets, 107, 2931 (2006).

2. C. Kalbach, Z. Phys. A 283, 401 (1977) 
3. C. Kalbach, Phys. Rev. C 71, 034606 (2005)

4. A. Iwamoto, K. Harada, Phys. Rev. C 26(5), 1821 (1982)

5. P.G. Young et al., Los Alamos National Laboratory Report No. LA-12343-MS (1992)

6. E. Beták, J. Dobeš, Z. Phys. A 279, 319 (1976)

7. M.B. Chadwick, P.G. Young, D.C. George, Y. Watanabe, Phys. Rev. C 50(2), 996 (1994)

8. S. Kunieda, S. Chiba, K. Shibata, A. Ichihara, E.Sh. Soukhovitskĩ̃, J. Nucl. Sci. Technol. 44(6), 838 (2007)

9. M. Avrigeanu, A.C. Obreja, F.L. Roman, V. Avrigeanu, W. von Oertzen, At. Data Nucl. Data Tables 95, 501 (2009)

10. M. Avrigeanu, V. Avrigeanu, Phys. Rev. C 79, 027601 (2009)

11. A.J. Koning, M.C. Duijvestijn, Nucl. Phys. A744, 15 (2004)

12. A.V. Ignatyuk, G.N. Smirenkin, A.S. Tishin, Sov. J. Nucl. Phys. 21, 255 (1975)

13. C. Kalbach, Z. Phys. A 287, 319 (1978)

14. N. Nica, S. Benck, E. Raeymackers, I. Slypen, J.P. Meulders, V. Corcalciuc, J. Phys. G: Nucl. Part. Phys. 28, 2823 (2002)

15. Z. Lewandowski, E. Loeffler, R. Wagner, H.H. Mueller, W. Reichart, P. Schober, E. Gadioli, E. Gadioli Erba, Lettere al Nuovo Cimento 28, 15 (1980)

16. K. Ammon, I. Leya, B. Lavielle, E. Gilabert, J.C. David, U. Herpers, R. Michel, Nucl.Instrum.Methods Phys.Res. B266, 2 (2008)

17. B. Piskor-Ignatowicz, F. Goldenbaum (2009), data are available from EXFOR

18. C. Kalbach, Phys. Rev. C37, 2350 (1988)

19. A.A. Cowley, G.J. Arendse, J.W. Koen, W.A. Richter, J.A. Stander, G.F. Steyn, P. Demetriou, P.E. Hodgson, Y. Watanabe, Phys. Rev. C 54(2), 778 (1996) 